\title{
Influência de aceptores de elétrons na ocorrência e desempenho de organismos acumuladores de fósforo em reator em batelada sequencial
}

\section{Influence of electron acceptors on the occurrence and performance of phosphorus accumulating organisms in sequencing batch reactor}

\author{
Data de entrada: \\ $01 / 11 / 2019$ \\ - Data de aprovação: \\ 06/08/2020
}

Rosângela Maria da Silva ${ }^{1 *}$ | Elivânia Vasconcelos Moraes dos Santos² | José Sérgio dos Santos ${ }^{3}$ | Hugo Leonardo de Brito Buarque ${ }^{3}$ | José Capelo Neto ${ }^{1}$

DOI: https://doi.org/10.36659/dae.2022.003

ORCID ID

Silva RM (DD https://orcid.org/0000-0001-5660-8187

Santos EVM (D) https://orcid.org/0000-0002-9690-4754

\author{
Santos JS (D) https://orcid.org/0000-0002-6201-0218 \\ Buarque HLB (D) https://orcid.org/0000-0002-8965-3846 \\ Capelo Neto J (D) https://orcid.org/0000-0002-7669-7153
}

\section{Resumo}

Os processos de remoção biológica de fósforo no tratamento de efluentes vêm ganhando espaço no Brasil e em outros países. Os principais responsáveis pela remoção de fósforo nesses processos são os organismos acumuladores de polifosfato. Alguns desses organismos também são capazes de utilizar nitrato ou nitrito, em vez de oxigênio, como aceptor de elétrons e, assim, desfosfatar e desnitrificar simultaneamente. Nesse contexto, este estudo avaliou o uso de diferentes aceptores de elétron no desenvolvimento de organismos acumuladores de polifosfato em reatores em batelada sequencial sob condições operacionais controladas, como também a influência desses aceptores no desempenho destes sistemas na remoção de matéria orgânica e de nutrientes a partir de efluentes aquosos. Então, três reatores em batelada sequencial com características favoráveis à ocorrência de organismos acumuladores de polifosfato foram operados com efluente sintético, utilizando oxigênio, nitrito ou nitrato como aceptor de elétrons. A idade do lodo (15 dias), a temperatura $\left(<20^{\circ} \mathrm{C}\right)$ e o $\mathrm{pH}(\sim 7,5)$ do meio foram definidos e controlados para favorecer o desenvolvimento desses organismos. O efluente sintético foi constituído por acetato de sódio $\left(900 \mathrm{mg} \mathrm{L}^{-1}\right)$, como substrato orgânico, além de macro e micronutrientes. Análises físicas e químicas (e.g., demanda química de oxigênio), ensaios respirométricos e testes estatísticos foram usados para avaliar a influência dos aceptores de elétrons na ocorrência dos organismos acumuladores de polifosfato e no desempenho dos reatores biológicos. As condições e estratégias operacionais usadas nos três reatores produziram licores mistos constituídos por organismos acumuladores de polifosfato e com concentrações médias de sólidos suspensos voláteis e totais variando entre 545 e $723 \mathrm{mg} \mathrm{L}^{-1}$ e entre 705 e $1.078 \mathrm{mg} \mathrm{L}^{-1}$, respectivamente. As médias das taxas máximas de consumo de oxigênio dos licores, obtidas nos testes respirométricos com pulsos de acetato e de propionato, variaram entre $60 \mathrm{e}$ $82 \mathrm{mg} \mathrm{L}^{-1} \mathrm{~h}^{-1}$ e entre 27 e $37 \mathrm{mg} \mathrm{L}^{-1} \mathrm{~h}^{-1}$, respectivamente. Os reatores diminuíram em mais de $85 \%$ a demanda

\footnotetext{
1 Universidade Federal do Ceará (UFC) - Fortaleza - Ceará - Brasil.

${ }^{2}$ Instituto Federal de Educação, Ciência e Tecnologia (IFCE) - Limoeiro do Norte - Ceará - Brasil.

${ }^{3}$ Instituto Federal de Educação, Ciência e Tecnologia (IFCE) - Fortaleza - Ceará - Brasil.

* Autora correspondente: rosangela_ifceahotmail.com.
} 
química de oxigênio e removeram mais de $70 \%$ do ortofosfato do efluente. $O$ reator com nitrato como aceptor de elétrons foi o menos eficiente na remoção de fósforo, bem como apresentou o maior teor de lodo produzido, o que pode representar custos adicionais ao processo. Os reatores que usaram oxigênio e nitrito foram equivalentes em desempenho (remoção de matéria orgânica e de fósforo) e na produção de biomassa.

Palavras-chave: Aceptores de elétrons. Organismos acumuladores de polifosfato. Reator em Batelada Sequencial. Remoção de fósforo. Respirometria.

\section{Abstract}

The enhanced biological phosphorus removal process in wastewater treatment plants has been gaining ground in Brazil and in other countries worldwide. The microorganisms largely responsible for phosphorus removal in this process are known as the polyphosphate accumulating organisms. Some these organisms have the ability to utilize nitrate or nitrite instead of oxygen as electron acceptors and, therefore, perform phosphorous uptake and denitrification simultaneously. In this context, this study evaluated the use of different electron acceptors in the development of polyphosphate accumulating organisms in sequential batch reactors under controlled operating conditions, as well as the influence of these acceptors on the performance these systems in the removal of organic matter and nutrients from aqueous effluents. Thus, three sequential batch reactors with characteristics suitable for the occurrence of phosphorus accumulating organisms were operated with synthetic effluent and using oxygen, nitrite or nitrate as an electron acceptor. The sludge age (15 days), temperature (below $20^{\circ} \mathrm{C}$ ) and pH $(\sim 7.5)$ of the medium were defined and controlled to promote the development of these organisms. The synthetic effluent was composed of sodium acetate $\left(900 \mathrm{mg} \mathrm{L}^{-1}\right)$ as organic substrate and macro and micronutrients. Physical and chemical analyzes (e.g., chemical oxygen demand), respirometry and statistical tests were used to evaluate the influence of electron acceptors on the occurrence of polyphosphate accumulating organisms and on the performance of the biological reactors. The operating conditions and strategies used in the reactors produced mixed liquors constituted of polyphosphate accumulating organisms and average concentrations of volatile and total suspended solids were varied from 545-723 mg L-1 and 705-1078 $\mathrm{mg} \mathrm{L}^{-1}$ respectively. The mean of the maximum rates of oxygen consumption of the liquors, obtained in the respirometry tests with pulses of acetate and propionate, varied from 60-82 $\mathrm{mg}^{\mathrm{L}-1} \mathrm{~h}^{-1}$ and 27-37 $\mathrm{mg} \mathrm{L}^{-1} \mathrm{~h}^{-1}$, respectively. The reactors reduced the chemical oxygen demand by more than $85 \%$ and removed more than $70 \%$ of the orthophosphate from the effluent. The reactor with nitrate as electron acceptor was the least efficient in removing phosphorus, as well as has produced the highest sludge content, which may represent additional costs to the process. The reactors using oxygen and nitrite have shown equivalent performance in the removal of organic matter and phosphorus and in the production of biomass.

Keywords: Electron acceptors. Polyphosphate accumulating organisms. Phosphorus removal. Respirometry. Sequencing Batch Reactor.

\section{INTRODUÇÃO}

Um dos principais problemas associados ao lançamento indiscriminado de águas residuárias sem o devido tratamento nos corpos hídricos é a eutrofização, particularmente devido à presença de nitrogênio e de fósforo (GE et al., 2018; LEE et al., 2018; LI et al., 2019). Esses nutrientes provo- cam a degradação do corpo hídrico quando dispostos no ambiente aquático em concentrações elevadas, já que permitem a proliferação excessiva de autótrofos, particularmente algas (FERREIRA, 2014; WANG, LIU, ZHENG, 2013). Além disso, o fósforo é comumente considerado o fator limitante para a eutrofização, já que o nitrogênio 
pode ser captado da atmosfera por meio de alguns tipos de bactérias e outros tipos de organismos procariontes, como as cianobactérias (LI et al., 2019).

Sistemas convencionais de lodo ativado conseguem remover apenas uma pequena fração do fósforo, incorporando-o no lodo, mas deixando ainda uma elevada concentração no efluente tratado. Zaman et al. (2019) relatam que o processo tradicional de remoção de nutrientes dificilmente atende aos padrões de descarga mais críticos. Sendo assim, a modificação dos sistemas convencionais pode resultar no aumento significativo da remoção desse elemento, tal como ocorre no sistema tipo UCT e em variantes do reator de batelada sequencial. Nesse sentido, processos de remoção biológica avançada de fósforo vêm ganhando espaço no Brasil e também em outros países (BOWES et al., 2015; COAT et al., 2011; MAHARDIKA, PARK, CHOO, 2018; ZAMAN et al., 2019; MELQUUIADES et al., 2017).

Li et al. (2019) explicam que a principal população bacteriana responsável pela remoção biológica do fósforo é a dos organismos acumuladoras de polifosfato. Ademais, um subgrupo dessa microbiota, os organismos acumuladores de polifosfato desnitrificantes (DPAO), além de removerem fósforo, utilizando o mesmo substrato orgânico, também removem nitrogênio, além de produzir menores teores de lodo de excesso (BASSIN et al., 2012; RANJAN et al., 2017). Wang et al. (2015) comentam que a remoção de fósforo utilizando os DPAO se baseia no uso de nitrato e nitrito (DPAO I) ou exclusivamente de nitrito (DPAO II) como aceptores de elétrons, diferentemente dos demais acumuladores de polifosfato (PAO), que utilizam o oxigênio. Assim, os DPAO podem ser mais eficientes no tratamento de efluentes que os PAO, já que removem simultaneamente fósforo e nitrogênio, além de minimizar os custos do processo (LIU et al., 2015).
É importante mencionar que as condições operacionais do tratamento, tais como o tipo de aceptor de elétrons, a temperatura e o $\mathrm{pH}$, devem garantir a eliminação ou minimização de grupos competidores pelo substrato disponível, como os organismos acumuladores de glicogênio (GAO), e que podem tornar limitada a remoção de fósforo (BASSIN et al., 2012; BUNCE et al., 2018; COSTA, 2019; CYDZIK-KWIATKOWSKA, ZIELINSKA, 2016; ZHAO et al., 2019).

Nesse contexto, este trabalho objetivou avaliar o uso dos aceptores de elétrons (oxigênio, nitrito e nitrato) no desenvolvimento de organismos acumuladores de polifosfato em sistemas de reatores em batelada sequencial (RBS) sob condições adequadas e controladas de temperatura, $\mathrm{pH}$ e idade de lodo, como também a influência destes aceptores no desempenho destes sistemas na remoção de matéria orgânica e nutrientes de efluentes, usando parâmetros e técnicas físico-químicas e respirométricas.

\section{METODOLOGIA}

\subsection{Reatores biológicos}

Neste estudo, foram operados três reatores de batelada sequencial (RBS) de câmara única, em escala laboratorial, construídos com recipientes tronco-piramidais de plástico transparente com volume total de 4,75 litros. Os reatores se diferenciavam na estratégia usada para favorecer a predominância no lodo de determinados subgrupos de bactérias acumuladoras de polifosfato, em detrimento dos demais subgrupos e de grupos microbianos competidores, como os GAO, seguindo recomendações de Oehmen et al. (2007) e Santos (2014).

Assim, o primeiro reator (RBS1) apresentava fases anaeróbia e aeróbia com presença de oxigênio gasoso, favorecendo o predomínio dos PAO; o segundo reator (RBS2) consistia de um sistema 
anaeróbio-anóxico com presença de nitrito $\left(\mathrm{NO}_{2}{ }^{-}\right)$, favorecendo a preponderância dos subgrupos DPAO I e DPAO II; enquanto o terceiro reator (RBS3) era constituído por um sistema anaeróbio-anóxico com presença de nitrato $\left(\mathrm{NO}_{3}^{-}\right)$, no qual podem predominar somente os DPAO I. Um esquema do funcionamento dos reatores utilizados, ilustrando os ciclos operacionais e seus respectivos tempos de operação, é apresentado na Fig. 1. O tempo de duração do ciclo completo de cada RBS foi de pouco mais de 6 horas. Os sistemas eram controlados por automatização estática utilizando temporizadores e bombas dosadoras peristálticas de vazão máxima nominal de $20 \mathrm{~L} / \mathrm{h}$.

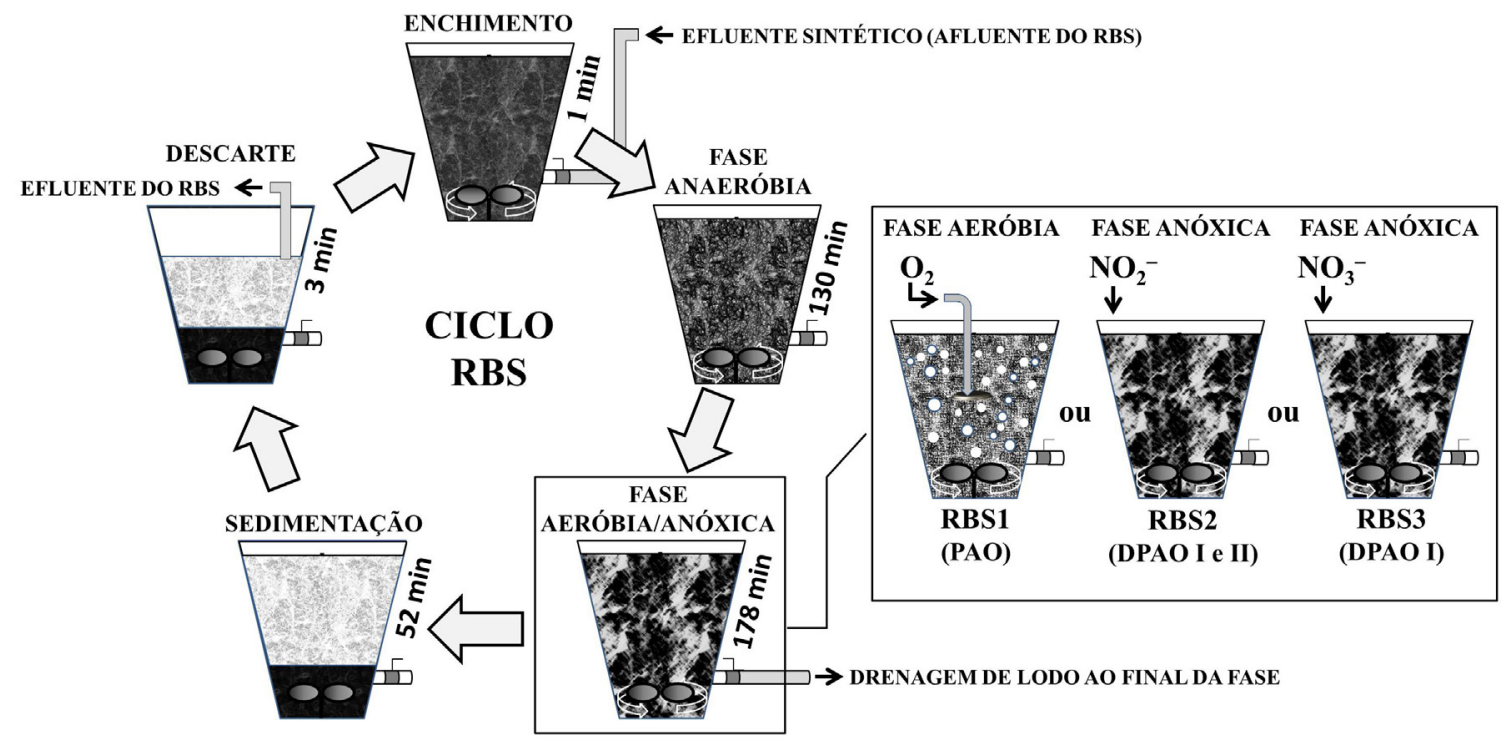

Figura 1 - Esquema de funcionamento dos reatores de batelada sequencial utilizados.

Na fase aeróbia do RBS1, os teores de oxigênio dissolvido (OD) chegavam a valores próximos do limite de saturação do meio, por intermédio do fornecimento de ar através de um sistema compressor (inalador comercial), que borbulhava o gás através de um difusor (pedra porosa) colocado no interior do reator. As fases anóxicas nos outros dois reatores eram obtidas por meio da adição de $150 \mathrm{~mL}$ de uma solução de nitrito de sódio ou de nitrato de sódio ao meio reacional, de modo a manter dosagens iniciais de $60 \mathrm{mg}-\mathrm{N} \mathrm{L}^{-1}$ de $\mathrm{NO}_{2}$, no RBS2, e de $\mathrm{NO}_{3}{ }^{-}$, no RBS3, as quais foram definidas seguindo os estudos de Santos (2014). $\mathrm{Na}$ fase anaeróbia dos reatores, as concentrações de OD permaneceram próximas de zero. A agitação de 50 rpm nas etapas de mistura e reação (fase anaeróbia e fase aeróbia ou anóxica) para suspensão do lodo era obtida com os impelidores de tur- bina de duas pás retas $90^{\circ}$ de um teste de jarros da marca Policontrol, modelo Turb-Floc/2C.

Além disso, o efluente sintético utilizado como substrato, o inóculo de partida e as demais condições operacionais dos reatores também foram estabelecidas com o intuito de fazer predominar no lodo as bactérias acumuladoras de polifosfato.

O efluente sintético alimentado nos reatores foi preparado com acetato de sódio, como substrato orgânico, e com sais básicos de fosfato de potássio, como fontes de fósforo. Os teores de referência destes e dos demais constituintes do efluente foram definidos com base na literatura (OEHMEN et al., 2006a; OEHMEN et al., 2007; SMOLDERS et al., 1994; SANTOS, 2014) e estão mostrados no Quadro 1. 
Quadro 1 - Composição do efluente sintético.

\begin{tabular}{|c|c|c|c|c|c|}
\hline $\mathrm{CH}_{3} \mathrm{COONa}$ & $900 \mathrm{mg} \mathrm{L}^{-1}$ & EDTA & $40 \mathrm{mg} \mathrm{L}^{-1}$ & $\mathbf{K I}$ & $0,64 \mathrm{mg} \mathrm{L}^{-1}$ \\
\hline $\mathrm{NH}_{4} \mathrm{Cl}$ & $1.020 \mathrm{mg} \mathrm{L}^{-1}$ & Levedura & $10 \mathrm{mg} \mathrm{L}^{-1}$ & $\mathbf{H}_{3} \mathrm{BO}_{3}$ & $0,60 \mathrm{mg}^{-1}$ \\
\hline $\mathrm{MgSO}_{4}$ & $586 \mathrm{mg} \mathrm{L}^{-1}$ & Peptona & $10 \mathrm{mg} \mathrm{L}^{-1}$ & $\mathrm{MnCl}_{2}$ & $0,36 \mathrm{mg} \mathrm{L}^{-1}$ \\
\hline $\mathrm{CaCl}_{2}$ & $144 \mathrm{mg} \mathrm{L}^{-1}$ & N-alil-tioureia & $5,0 \mathrm{mg} \mathrm{L}^{-1}$ & $\mathrm{CoCl}_{2}$ & $0,33 \mathrm{mg} \mathrm{L}^{-1}$ \\
\hline $\mathrm{K}_{2} \mathrm{HPO}_{4}$ & $130 \mathrm{mg} \mathrm{L}^{-1}$ & $\mathrm{FeCl}_{3}$ & $3,6 \mathrm{mg} \mathrm{L}^{-1}$ & $\mathrm{ZnSO}_{4}$ & $0,27 \mathrm{mg} \mathrm{L}^{-1}$ \\
\hline $\mathrm{KH}_{2} \mathrm{PO}_{4}$ & $100 \mathrm{mg} \mathrm{L}^{-1}$ & & & $\mathrm{CuSO}_{4}$ & $0,08 \mathrm{mg} \mathrm{L}^{-1}$ \\
\hline
\end{tabular}

A concentração utilizada do acetato resultou numa demanda química de oxigênio (DQO) e numa concentração de carbono orgânico aproximadamente iguais a $700 \mathrm{mg}-\mathrm{O}_{2} \mathrm{~L}^{-1}$ e $265 \mathrm{mg}-\mathrm{C} \mathrm{L}^{-1}$, respectivamente. Também é importante mencionar que o baixo valor da razão obtida entre o teor de carbono

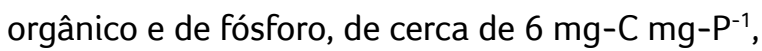
favorece o crescimento das bactérias acumuladoras de polifosfato (OEHMEN et al., 2007).

O inóculo de partida utilizado foi previamente aclimatado durante 30 dias num RBS com volume útil de 4 litros, sendo submetido a condições anaeróbias-aeróbias e temperatura inferior a $20^{\circ} \mathrm{C}$. Esse limite de temperatura foi obtido pelo sistema de condicionamento de ar do ambiente laboratorial e colabora no predomínio das bactérias acumuladoras de polifosfato (PANSWAD, DOUNGCHAI, ANOTA, 2003). O afluente desse reator consistia de uma solução com a mesma composição apresentada no Quadro 1 e mantido em $\mathrm{pH}$ próximo de 7,0 por meio da adição de $\mathrm{HCl}$ $0,5 \mathrm{~mol} \mathrm{~L}^{-1}$ ou de $\mathrm{NaOH} \mathrm{0,5} \mathrm{mol} \mathrm{L}^{-1}$, tendo em vista que as bactérias acumuladoras de polifosfato têm melhores desempenhos em meio neutro, como também não suportam $\mathrm{pH}$ menores que 5,2 e maiores que 9,5 (STENSEL, 1991; OEHMEN et al., 2005). Um volume de 2 litros do afluente era realimentado diariamente no reator, logo após o descarte de igual volume da suspensão sobrenadante resultante da etapa de sedimentação. $O$ sistema foi operado de modo a oferecer uma idade de lodo de 10 dias (drenagem de 400 $\mathrm{mL} /$ dia de lodo).
A partida de cada um dos três reatores avaliados se deu pela adição inicial de $400 \mathrm{~mL}$ do licor misto (inóculo de partida) previamente aclimatado, o qual apresentou teor de sólidos suspensos voláteis (SSV) aproximadamente igual a $500 \mathrm{mg} \mathrm{L}^{-1}$, seguindo-se da adição de um volume suficiente do efluente sintético para completar 4 litros em cada RBS. A temperatura e o $\mathrm{pH}$ do meio foram mantidos da mesma forma e nos mesmos intervalos de valores utilizados na aclimatação do inóculo de partida pelos motivos já apresentados.

A partir de então, em cada ciclo operacional de cada reator, eram drenados $70 \mathrm{~mL}$ do licor misto (mantendo o lodo com idade de 15 dias), logo após a etapa aeróbia/anóxica, bem como o líquido sobrenadante residual da etapa de sedimentação era descartado: cerca de 1,0 litro a partir do RBS1 e 1,2 litros a partir do RBS2 e do RBS3. Os $0,2 \mathrm{~L}$ adicionais no descarte desses dois últimos reatores se dava para compensar a adição das soluções de nitrito e de nitrato no início da fase anóxica. Ademais, o efluente sintético era alimentado na fase de enchimento (começo de cada ciclo dos reatores), de modo completar o volume do meio até o valor de 4 litros.

\subsection{Avaliação das características e da eficiência dos sistemas estudados}

Análises físicas e químicas e testes respirométricos foram conduzidos com o intuito de avaliar o desempenho e a estabilidade dos três reatores estudados. Assim, para cada reator em distintos ciclos operacionais aleatoriamente selecionados, amostras de afluente (efluente sintético), 
de licor misto, e ou de correntes efluentes foram coletadas e analisadas, como também foram colocados instrumentos de medição no meio reacional para monitorar os sistemas avaliados.

As variáveis físicas e químicas monitoradas e analisadas foram: DQO; teores de nitrato $\left(\mathrm{NO}_{3}{ }^{-}\right)$, de nitrito $\left(\mathrm{NO}_{2}{ }^{-}\right)$e de ortofosfato $\left(\mathrm{PO}_{4}{ }^{3-}\right)$; $\mathrm{OD} ; \mathrm{pH}$; temperatura; teores de sólidos suspensos totais (SST) e de sólidos suspensos voláteis (SSV). Essas variáveis com suas respectivas unidades, métodos e princípios analíticos utilizados estão apresentados no Quadro 2.

Quadro 2 - Variáveis e métodos analíticos utilizados para os sistemas operados

\begin{tabular}{|c|c|c|c|}
\hline Variável & Unidade & Método(*) & Princípio Analítico \\
\hline DQQO & $\mathrm{mg}-\mathrm{O}_{2} \mathrm{~L}^{-1}$ & $5220 \mathrm{C}$ & Oxidação em refluxo fechado e titrimetria \\
\hline $\mathrm{NO}_{3}^{-}$ & $\mathrm{mg}-\mathrm{NO}_{3}^{-}-\mathrm{NL}^{-1}$ & $4500-\mathrm{NO}_{3}^{-} \mathrm{C}$ & Varredura no ultravioleta \\
\hline $\mathrm{NO}_{2}^{-}$ & $\mathrm{mg}-\mathrm{NO}_{2}{ }^{-}-\mathrm{NL}^{-1}$ & $4500-\mathrm{NO}_{2}^{-} \mathrm{B}$ & Colorimetria após diazotação \\
\hline $\mathrm{OD}$ & $\mathrm{mg}-\mathrm{O}_{2} \mathrm{~L}^{-1}$ & 45000 & Eletrometria \\
\hline $\mathrm{PO}_{4}^{-}$ & $\mathrm{mg}-\mathrm{PO}_{4}^{3-}-\mathrm{PL}^{-1}$ & $4500-P E$ & Colorimetria após digestão \\
\hline $\mathrm{pH}$ & - & $4500-\mathrm{H}^{+} \mathrm{B}$ & Eletrometria \\
\hline SST & $\mathrm{mg} \mathrm{L}^{-1}$ & $2540 \mathrm{D}$ & Gravimetria \\
\hline SSV & $\mathrm{mg} \mathrm{L}^{-1}$ & $2540 \mathrm{E}$ & Gravimetria após filtração em membrana \\
\hline Temperatura & ${ }^{\circ} \mathrm{C}$ & $2550 \mathrm{~B}$ & Termometria de mercúrio \\
\hline
\end{tabular}

Nota: (*) De acordo com o Standard Methods for the Examination of Water and Wastewater (APHA, 2017).

O teste respirométrico é uma técnica rápida, simples e prática, que também permite avaliar a existência dos PAO, bem como fazer análises comparativas deste grupo de organismos em relação a outros grupos competidores (e.g., GAO) de acordo com cada característica e condições operacionais nos sistemas biológicos. Assim, ensaios respirométricos foram conduzidos num respirômetro, da marca Beluga, de acordo com o protocolo descrito em Van Haandel e Catunda (1982) e, posteriormente, em outros estudos (CATUNDA et al., 1996; DERKS, 2007; SILVA FILHO, 2009; MIRANDA, 2012; SANTOS, 2014).

As taxas de consumo de oxigênio (TCO) utilizando pulsos de acetato de sódio e de propionato de cálcio como substratos, nas concentrações de

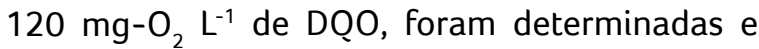
avaliadas em amostras de $1 \mathrm{~L}$ de licor misto coletadas nos mesmos ciclos selecionados para algumas análises de sólidos suspensos totais e voláteis, após a fase anaeróbia desses ciclos. Então, parâmetros cinéticos e estequiométricos foram determinados e utilizados na obtenção dos va- lores máximos de TCO após os pulsos de acetato $\left(\mathrm{TCO}_{\mathrm{HAC}}\right)$ e de propionato $\left(\mathrm{TCP}_{\mathrm{HPr}}\right)$, os quais podem ser utilizados na avaliação dos grupos de organismos existentes no lodo dos reatores estudados.

A escolha dos substratos foi baseada nos trabalhos de Melquiades et al. (2017); Santos (2014); e Oehmen et al. (2006a), que demonstram que ambos os substratos são bem assimilados pelos organismos acumuladores de polifosfato e permitem avaliar a existência e competição de grupos de GAO nos sistemas biológicos de remoção de fósforo. $A$ temperatura nesses testes não ultrapassou $20^{\circ} \mathrm{C}$, um diferencial deste estudo, de modo a manter no teste que as mesmas características operacionais usadas nos reatores estudados. Os valores de $\mathrm{pH}$ eram verificados antes do teste e mantidos entre 7,0 e 8,0 com esse mesmo intuito.

\subsection{Avaliação da predominância de organismos acumuladores de polifosfato}

Uma opção para avaliação da existência e predomínio dos organismos acumuladores de polifos- 
fato seria a técnica de biologia molecular. Porém, diante das dificuldades e do alto custo dessa técnica, foram utilizadas duas estratégias que, discutidas por Santos (2014) e por Melquiades et al. (2017), podem ser ferramentas de correlação da existência e predominância desses organismos por meio de parâmetros físico-químicos e de dados de respirometria.

Nesse sentido, as frações de voláteis nos sólidos suspensos, determinada com a razão entre o teor de SSV e SST, aqui denominada razão S, como também as razões obtidas pelo quociente entre $\mathrm{TCO}_{\mathrm{HAc}}$ e $\mathrm{TCO}_{\mathrm{HPr}}$, denominada razão T, foram calculadas para as amostras de licor misto dos sistemas RBS1, RBS2 e RBS3 e utilizadas como ferramentas de avaliação da predominância do organismos acumuladores de polifostato nesses sistemas.

Os valores médios da razão $\mathrm{S}$ e da razão $\mathrm{T}$ determinados nos ensaios respirométricos, a $20^{\circ} \mathrm{C}$ e pH entre 7,0 e 8,0, com os licores coletados nos reatores RBS1, RBS2 e RBS3 foram, então, analisados seguindo os critérios discutidos em Santos (2014).

\subsection{Determinação de constantes cinéticas do processo de remoção de fósforo}

A respirometria também pode ser usada, quando aliada a um bom modelo matemático, na estimativa de coeficientes estequiométricos e de taxas metabólicas dos organismos presentes no lodo de um reator biológico (SANTOS, 2014). Neste estudo, taxas específicas de consumo de oxigênio (TCOe) foram obtidos a partir dos ensaios respirométricos e os valores médios obtidos foram analisados com o intuito de comparar a resposta metabólica dos lodos dos três reatores avaliados em função do acetato e do propionato como substrato dos sistemas.

\subsection{Análise Estatística}

Diferentes técnicas estatísticas foram utilizadas na análise dos dados produzidos neste estudo. Inicialmente, a normalidade (distribuição) de cada subconjunto de dados foi avaliada pelo teste de Shapiro-Wilk (FIELD, 2020). Além disso, diversas estatísticas descritivas foram calculadas e apresentadas de forma tabular ou gráfica, tais como médias, desvios padrões, resumo dos cinco números, utilizando o programa estatístico SPSS (versão 21.0) nos cálculos estatísticos e o programa de tratamento gráfico Origin 9.1 para apresentação gráfica dos dados.

Ademais, intervalos de confiança a $95 \%$ e testes de inferência paramétricos e não paramétricos, tais como testes de hipóteses e de comparação de médias, também foram determinados e analisados com o intuito de avaliar o desempenho dos RBS estudados e respectivos fatores intervenientes.

Os intervalos de confiança a $95 \%$, particularmente, foram determinados considerando uma distribuição t-Student, como apresentado em Triola (2017), utilizando o programa IBM SPSS, ou a partir do teste de Wilcoxon, utilizando o Programa R (versão 4.0.0), como discutido por Geyer (2003).

Os demais testes de inferência foram conduzidos no IBM SPSS de acordo com a suposição de normalidade e de heterogeneidade atribuída aos dados (FIELD, 2020), todos considerando nível de confiança de $95 \%$ ou significância de $5 \%$. Assim, testes t para uma ou duas amostras, análise de variância (ANOVA) e teste de comparações múltiplas de Bonferroni foram aplicados aos subconjuntos de dados indicados como seguindo uma distribuição normal pelo teste de Shapiro-Wilk, enquanto testes de Kruskal-Wallis, Mann-Whitney e de Kolmogorov-Smirnov foram usados nos subconjuntos em que algum conjunto não seguiu essa distribuição. 


\section{RESULTADOS E DISCUSSÕES}

\subsection{Desempenho dos sistemas RBS}

Os reatores RBS1, RBS2 e RBS3 foram operados simultaneamente e de modo a manter as condições mais adequadas para a remoção de fósforo ao longo de 150 dias. A temperatura e o $\mathrm{pH}$ dos três sistemas foram mantidos, respectivamente, em $18 \pm 2^{\circ} \mathrm{C}$ e em 7,5 $\pm 0,5$ praticamente por todo o período de operação. O OD dos reatores foi monitorado com valores inferiores a $0,6 \mathrm{mg}-\mathrm{O}_{2} \mathrm{~L}^{-1}$, exceto no RBS1, onde o meio apresentou valores de OD de até 6,5 $\mathrm{mg}-\mathrm{O}_{2} \mathrm{~L}^{-1}$ durante a fase aeróbia dos ciclos.
Diagramas boxplot para os valores de SST e SSV e para os valores máximos de TCO, nos pulsos com acetato $\left(\mathrm{TCO}_{\mathrm{HAc}}\right)$ e com propionato $\left(\mathrm{TCO}_{\mathrm{HPr}}\right)$, determinados para licor misto (do final da fase anaeróbia) dos três reatores estudados são apresentados na Fig. 2. Apesar da elevada amplitude apresentada nos valores dos sólidos, convém ressaltar que os dados são do lodo dos reatores e que admitem flutuações, pois acompanham a evolução adaptativa da microbiologia dos sistemas.
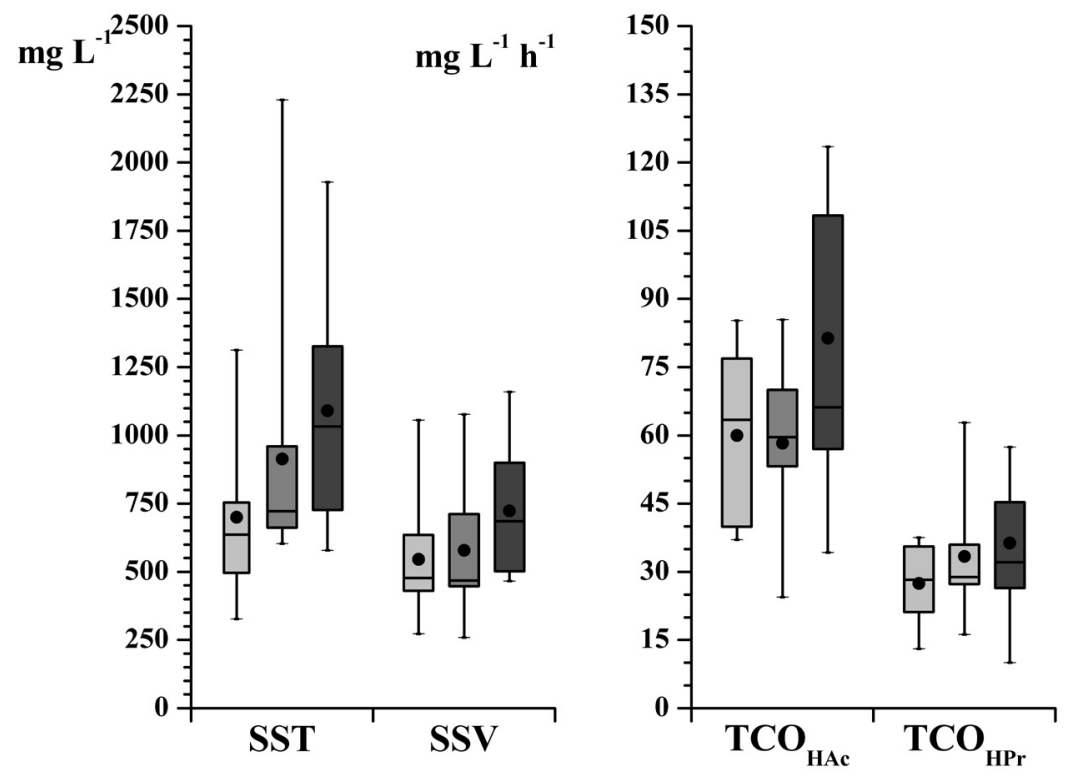

\section{Legenda: $\square$ RBS1 $\square$ RBS2 $\square$ RBS3}

Figura 2 - Diagramas boxplot dos teores de sólidos suspensos totais e voláteis e das taxas máximas de consumo de oxigênio (pulsos respirométricos de acetato e propionato) determinadas para amostras de licor misto dos três reatores avaliados neste estudo.

Nota: os círculos no interior das caixas correspondem à média dos valores da variável considerada.

Os dados de sólidos suspensos e de TCO dos sistemas podem caracterizar os agentes biológicos do tratamento e foram utilizados como ferramentas no estudo para acompanhamento do metabolismo (MELQUIADES et al., 2017; SANTOS, 2014). Destaca-se que, com mais sólidos em suspensão no sistema, maior é a eficiência do processo na remoção de DQO e de nutrientes, devido à aceleração metabólica (WANG et al. 2008). Os dados de sólidos variaram segundo a operação dos sistemas, o que não compromete a avaliação dos resultados, pois para cada teste de TCO é possível relacioná-lo com os sólidos, obtendo, assim, as TCO específicas. 
Os conjuntos de valores obtidos de sólidos suspensos e de taxas máximas de consumo de oxigênio para os licores mistos dos reatores estudados seguiram uma distribuição normal ( $p$-valor > 0,05), de acordo com os testes de Shapiro-Wilk realizados, excetuando-se o conjunto amostral de SST do RBS2 ( $p$-valor < 0,01). Por esse motivo, análises paramétricas foram empregadas na comparação entre os conjuntos de dados de SSV, $\mathrm{TCO}_{\mathrm{HAc}}$ e $\mathrm{TCO}_{\mathrm{HPr}}$ para os três reatores avaliados, conquanto testes não paramétricos foram usados com os dados de SST.

Os intervalos de confiança de $95 \%$ para os teores de SST nos reatores RBS1, RBS2 e RBS3, obtidos a partir de um teste de Wilcoxon (GEYER, 2003), são mostrados na Tabela 1. Observa-se uma tendência de aumento nos SST do RBS1 ao RBS3. Um teste de Kruskal-Wallis aplicado aos três conjuntos de dados de SST evidenciaram ( $p$-valor $<0,05$ ) haver pelo menos uma mediana populacional distinta das demais. Testes subsequentes de Kolmogorov-Smirnov para duas amostras revelaram que a diferença entre as medianas somente foi estatisticamente significativa ( $p$-valor $<0,05$ ) entre os sistemas RBS1 e RBS3, indicando que os teores de SST neste último reator são maiores que os observados no RBS1, corroborando a tendência de aumento observada.

Tabela 1- Intervalos de confiança de 95\% para os teores de sólidos suspensos e taxas máximas de consumo de oxigênio do licor misto dos reatores RBS1, RBS2 e RBS3.

\begin{tabular}{|c|c|c|c|c|}
\hline \multirow{2}{*}{ Reator } & \multicolumn{4}{|c|}{ Intervalo de confiança de $\mathbf{9 5} \%$} \\
\hline RBS1 & $\mathbf{S S T}\left(\mathbf{m g ~ L}^{-\mathbf{1}}\right)$ & SSV $\left(\mathbf{m g ~ L}^{-1}\right)$ & TCOHAc $\left(\mathbf{m g ~ L}^{-1} \mathbf{h}^{-1}\right)$ & $\operatorname{TCOHPr}\left(\mathbf{m g ~ L} \mathbf{~}^{-1} \mathbf{h}^{-1}\right)$ \\
\hline RBS2 & $705 \pm 179$ & $545 \pm 133$ & $60,00 \pm 13,03$ & $27,47 \pm 6,22$ \\
\hline RBS3 & $842 \pm 159$ & $579 \pm 142$ & $58,28 \pm 12,47$ & $33,37 \pm 9,21$ \\
\hline
\end{tabular}

Os intervalos de confiança de $95 \%$ para os teores de SSV e para $\mathrm{TCO}_{\mathrm{HAC}}$ e $\mathrm{TCO}_{\mathrm{HP}}$, nos reatores RBS1, RBS2 e RBS3, obtidos a partir de testes t (FIELD, 2020; TRIOLA, 2017), também são apresentados na Tabela 1. Entretanto, diferentemente do observado para os SST, as análises de variância (ANOVA) conduzidas com aquelas variáveis evidenciaram não haver diferença estatística ( $p$-valor $>0,05$ ) entre as respectivas médias populacionais de tais propriedades nos sistemas RBS1, RBS2 e RBS3.

A igualdade dos teores médios de SSV nos reatores indica que tais sistemas biológicos apresentavam praticamente a mesma concentração de lodo, haja vista que esses teores estão relacionados à quantidade de microrganismos presentes no meio. Os valores obtidos para esse parâmetro mostraram que os sistemas estiveram em confor- midade com os modelos previstos pela International Water Association, discutidos por Van Haandel e Marais (1999). A manutenção dos valores desses parâmetros foram cruciais para que os sistemas mantivessem as melhores condições, como a idade do lodo, para o crescimento dos microrganismos acumuladores de polifosfato.

Nos três reatores estudados foram observados valores consideráveis de $\mathrm{TCO}_{\mathrm{HPr}}$, confirmando a presença de organismos acumuladoras de polifosfato, em grande quantidade, visto que o propionato é um substrato quase exclusivo desses organismos acumuladores nesses sistemas. Ademais, conforme esperado, os valores amostrais de $\mathrm{TCO}_{\mathrm{HAc}}$ foram sempre maiores que os de $\mathrm{TCO}_{\mathrm{HPr}}$, haja vista o acetato ser substrato tanto dos acumuladores de polifosfato como dos GAO, que tipicamente estão pre- 
sentes em sistemas de lodo ativado. Santos (2014) indica que quanto mais a relação $\mathrm{TCO}_{\mathrm{HPr}} / \mathrm{TCO}_{\mathrm{HAc}}$ se aproxima de 1, maior é a presença (em termos de predominância, notadamente para culturas puras e semipuras) de organismos PAO no sistema, tendo a autora obtido valores aproximadamente de 0,30 para sistemas operados com esgoto sintético e matriz de esgoto sanitário contendo somente acetato; nessa pesquisa a relação esteve sempre acima de 0,40 para os três sistemas, chegando a quase 0,58 para RBS2.

Uma ANOVA revelou não haver diferença estatística ( $p$-valor $>0,05$ ) entre as médias populacionais de cada uma dessas taxas de consumo nos sistemas RBS1, RBS2 e RBS3, sugerindo que as condições operacionais estabelecidas nesses reatores propiciaram um lodo com características similares em termos de organismos acumuladores de polifosfato.

Em relação às demais variáveis de desempenho avaliadas, os valores médios de DQQ e dos teores de ortofosfato, de nitrito e de nitrato determinados para amostras coletadas das correntes afluente (efluente sintético) e efluente (sobrenadante descartado) desses reatores em 10 ciclos distintos e ao longo do período de operação estão representados nos diagramas de colunas mostrados na Fig. 3.

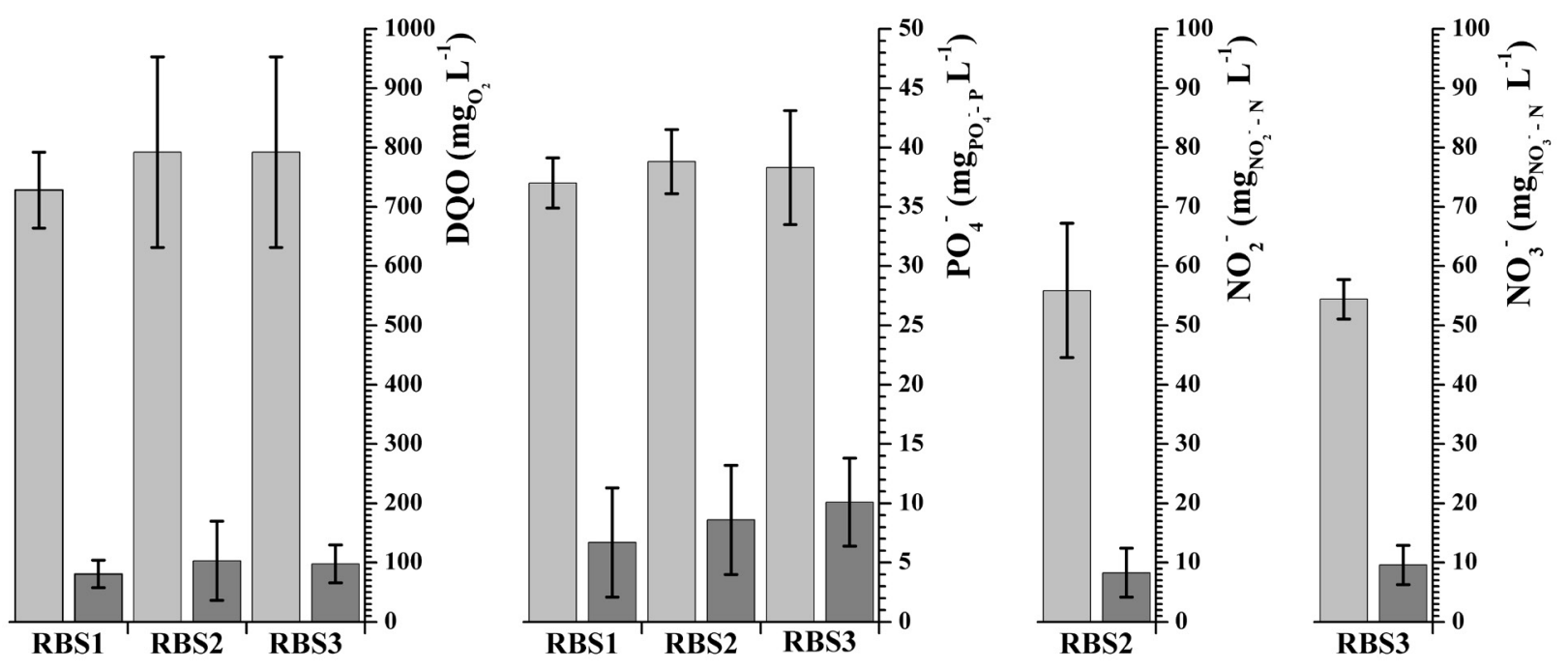

Legenda: $\square$ Afluente

Efluente

Figura 3 - Diagramas de colunas dos valores de demanda química de oxigênio e dos teores de ortofosfato, nitrito e nitrato determinados para as correntes afluente e efluente dos três reatores avaliados neste estudo. Nota: as barras de erro nos diagramas indicam o desvio padrão dos dados obtidos.

Os conjuntos de valores de DQO e de teores de ortofosfato total para as correntes afluente e efluente de cada um dos reatores estudados, na sua maioria, não seguiram uma distribuição normal ( $p$-valor $<0,05)$, de acordo com os testes de Shapiro-Wilk realizados. De maneira diferente, somente os teores finais de nitrito, nos ciclos do RBS2, não seguiram uma distribuição normal, também considerando um nível de significância de $5 \%$, indicando que testes paramétricos podem ser aplicados aos conjuntos de teores iniciais de nitrito e de nitrato, nos reatores RBS2 e RBS3, bem como aos de teores finais de nitrato no reator RBS3. 
Assim, testes de Kruskall-Wallis aplicados aos dados de DQO afluente e efluente revelam não haver evidência estatística ( $p$-valor $>0,05$ ) de diferenças entre as populações dessas variáveis nos distintos reatores avaliados, corroborando o fato de que os três reatores iniciaram os ciclos com uma mesma DQO e demonstrando que eles apresentaram uma mesma eficiência na remoção de matéria orgânica. Então, o intervalo de confiança da eficiência de redução da DQQ nos reatores foi estimado, a partir de um teste de Wilcoxon, como $88,5 \% \pm 1,5 \%$. Essa faixa de percentuais de remoção de DQO , maior que $80 \%$, foi condizente com o esperado para sistemas de lodo ativado (METCALF \& EDDY, 2016; VON SPERLING, 2007).

Em relação aos teores de ortofosfato total, os testes de Kruskall-Wallis para amostras independentes aplicados evidenciaram que os reatores avaliados tinham o mesmo teor inicial desse nutriente ( $p$-valor $>0,05)$, conquanto eles apresentaram diferenças estatisticamente relevantes ( $p$-valor $<0,05)$ entre os teores finais observados. Testes de Mann-Whitney usados na comparação de pares de reatores evidenciaram que a remoção de ortofosfato só foi significativa ( $p$-valor $<0,01$ ) entre o RBS1 e o RBS3, sugerindo que neste tipo de reator uma fase anóxica com excesso de nitrato pode desfavorecer a remoção de ortofosfatos quando comparada a uma fase aeróbia. De qualquer forma, os intervalos de confiança da eficiência de remoção de ortofosfato nos reatores RBS1, RBS2 e RBS3 foram estimados, a partir do teste de Wilcoxon, respectivamente, como $78,9 \% \pm 9,1 \%$, $77,1 \% \pm 10,2 \%$ e $73,3 \% \pm 5,9 \%$, as quais sugerem a existência de organismos acumuladores de polifosfato nos sistemas (LI et al., 2019; BASSIN et al., 2012) e que o uso de RBS na remoção deste nu- triente é viável quando as condições operacionais são adequadas ao processo.

Os resultados obtidos em relação aos teores de nitrito, no RBS2, e de nitrato, no RBS3, cujos intervalos de confiança a $95 \%$ dos teores iniciais foram, respectivamente, iguais a $54,55 \pm 2,55 \mathrm{mg}^{-N^{-1}} \mathrm{e}$ $55,86 \pm 8,65 \mathrm{mg}^{-\mathrm{N} \mathrm{L}^{-1}}$, isto é, com médias próximas ao valor de dosagem inicial proposto ( $60 \mathrm{mg}-\mathrm{N} \mathrm{L}^{-1}$ ), mostraram que houve reduções significativas dos teores destes aceptores de elétrons durante os ciclos desses dois reatores, cujos intervalos de confiança a $95 \%$ dos percentuais de remoção foram, respectivamente, de $85,5 \% \pm 3,2 \%$ e $82,2 \% \pm 5,0 \%$, para nitrito e nitrato. Essas elevadas remoções indicam a provável existência de grupos DPAO nos lodos desses reatores (BASSIN et al., 2012).

\subsection{Avaliação da predominância de organismos acumuladores de polifosfato}

A análise do desempenho dos reatores, particularmente no que diz respeito às remoções de matéria orgânica e de nutrientes (ortofosfato, nitrito e nitrato), demonstraram que as estratégias e condições adotadas neste estudo foram satisfatórias na obtenção dos bons resultados auferidos. Contudo, é também importante avaliar o papel do inóculo e do seu condicionamento nesses resultados, particularmente quanto à influência dos organismos acumuladores de polifosfato (PAO e DPAO) neste processo. Neste sentido, os valores médios e os desvios padrão da fração de voláteis nos sólidos suspensos e a razão entre os valores máximos de TCO após pulsos respirométricos de propionato e acetato, no licor misto do sistema, foram determinados e estão apresentados nos diagramas de linha da Fig. 4. 

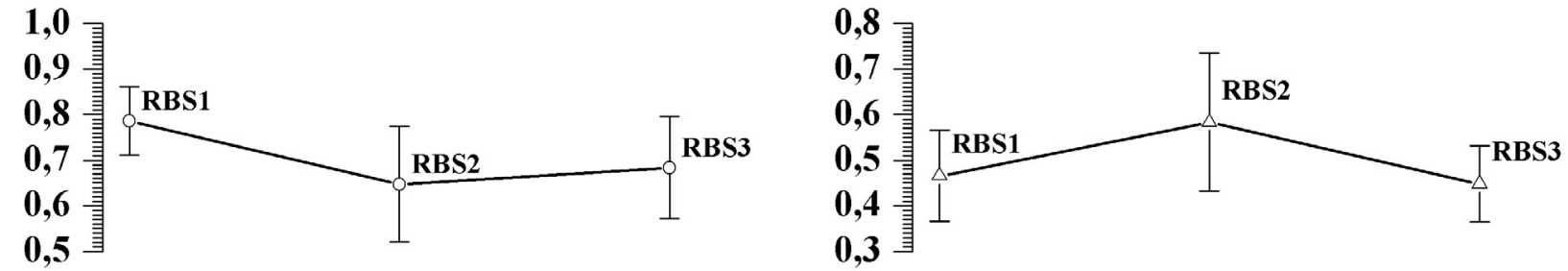

\section{Legenda: $-\circ-\operatorname{Razão} S-\triangle-\operatorname{Razão} T$}

Figura 4 - Diagramas de linha das frações médias de voláteis nos sólidos suspensos e das razões entre as taxas máximas de consumo de oxigênio com pulsos respirométricos de propionato e de acetato para amostras de licor misto dos sistemas RBS1, RBS2 e RBS3. As barras de erro representam os desvios padrão dos respectivos conjuntos de dados.

Os conjuntos de valores obtidos nas razões $\mathrm{S}$ e $\mathrm{T}$, nos testes respirométricos com os licores dos três reatores avaliados, seguiram uma distribuição normal ( $p$-valor > 0,05), de acordo com os testes de Shapiro-Wilk realizados. Por esse motivo, análises paramétricas foram empregadas com esses conjuntos de dados.

As frações de voláteis nos sólidos suspensos nas amostras analisadas variaram entre 0,43 a 0,93 com o predomínio de valores maiores que 0,70 ( $p$-valor $>0,05)$. A literatura tem, em geral, reportado valores médios da razão $S$ mais baixos (próximos de 0,46) para licores de RBS utilizados na remoção de fósforo, o que caracterizaria a predominância de organismos acumuladores de polifosfato (inorgânico) no lodo do sistema (SANTOS, 2014; VAN HAANDEL, MARAIS, 1999). Segundo os autores acima mencionados, valores próximos a 0,7 aparecem mais para lodos mistos com presença de diferentes grupos microbianos, ainda que com presença significativa de lodo PAO, mas sem a sua predominância.

Nos sistemas estudados, particularmente no RBS1, cuja média da razão $S$ foi de 0,79 , não se evidenciou ( $p$-valor < 0,05) o domínio dos acumuladores de polifosfato no lodo dos reatores, conquanto é possível afirmar que os sistemas biológicos avaliados, especialmente o RBS2 e o RBS3, têm características de um lodo misto (SANTOS, 2014) contendo PAO e ou DPAO em quantidades relevantes e que justificam as boas remoções obtidas tanto de DQO como de ortofosfato e demais nutrientes analisados.

Ademais, as razões entre $\mathrm{TCO}_{\mathrm{HPr}}$ e $\mathrm{TCO}_{\mathrm{HAc}}$ foram comparadas entre si e a um valor de referência $(1,0)$, considerando que quanto mais a razão $T$ se aproxima da unidade, maior a ocorrência e predominância de organismos acumuladores de polifosfato nos RBS (OEHMEN et al., 2006b; SANTOS, 2014). Assim, testes de hipóteses (testes t de uma amostra) comparando as médias amostrais da razão T com uma média populacional unitária indicaram que em nenhum reator estudado houve um domínio desses organismos acumuladores nos seus licores, visto que tais testes resultaram em $p$-valores menores que $5 \%$. Isso significa que outros grupos microbianos estão presentes em quantidade relevantes no lodo produzido, muito provavelmente acumuladores de glicogênio, os quais competem com os PAO e DPAO pelo substrato acético (BASSIN et al., 2012). De qualquer forma, esses resultados corroboram o inferido anteriormente: que os reatores foram operados com lodos mistos, constituídos, a priori, por GAO, PAO e ou DPAO, o que justifica os bons resultados obtidos de remoção de acetato (DQO) e de nutrientes, particularmente o ortofosfato.

É fácil notar que o valor médio da razão $T$ do RBS2 se sobressai entre os demais, com o valor 0,58 sugerindo que nesse reator há uma ocor- 
rência em maior proporção dos acumuladores de polifosfato em relação aos outros sistemas. Então, uma ANOVA conduzida com tais razões amostrais evidenciou haver alguma diferença estatística ( $p$-valor $<0,05)$ entre as médias populacionais de tais relações nos sistemas RBS1, RBS2 e RBS3. Testes de comparações múltiplas de Bonferroni foram, então, realizados e revelaram haver uma diferença significativa ( $p$-valor < $0,05)$ somente entre as médias da razão $T$ do licor do RBS2 e do RBS3, respectivamente, o maior e o menor valor médio obtidos.

Também é importante destacar que a menor média da razão T no RBS3 possivelmente retrata uma elevada proporção de GAO no licor desse reator, considerando que esse grupo de microrganismos é o principal competidor com PAO e DPAO pelo substrato (acetato) nesse tipo de sistema biológi- co (BASSIN et al., 2012; TAYÀ et al., 2013). Além disso, essa maior quantidade de GAO explicaria os maiores teores de SST neste reator, resultante de uma maior produção de lodo (TAYÀ et al., 2013), bem como os menores percentuais de desfosfatação do efluente sintético (BASSIN et al., 2012) alimentado neste reator comparativamente às remoções de fósforo nos outros dois sistemas.

\subsection{Testes respirométricos para avaliação do metabolismo microbiano do lodo}

Os valores médios e os desvios padrão das TCOe após pulsos respirométricos de acetato e de propionato, no licor misto dos sistemas estudados, com o intuito de avaliar o metabolismo dos organismos acumuladores presentes foram determinados e estão apresentados nos diagramas de linha da Fig. 5.
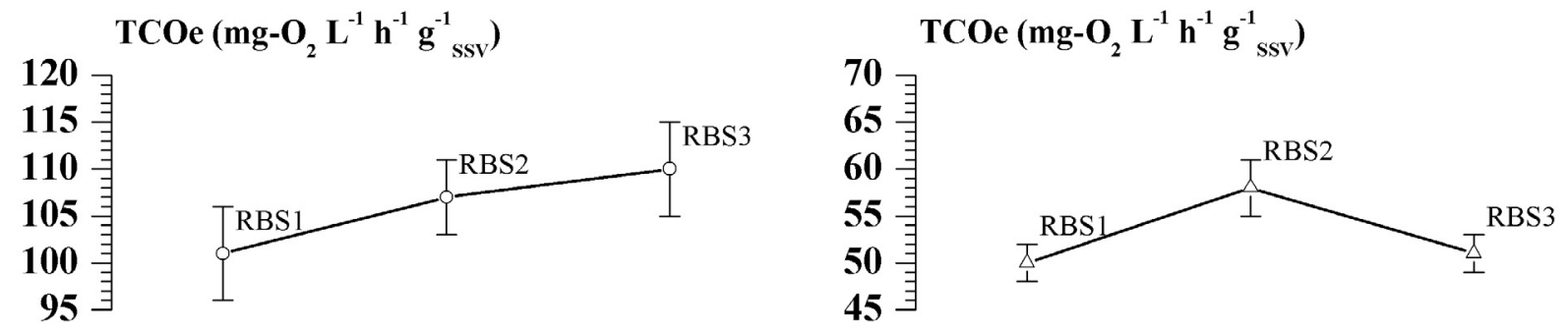

\section{Legenda: $-\circ-$ Acetato $-\triangle-$ Propionato}

Figura 5 - Diagramas de linha das taxas específicas médias de consumo de oxigênio com pulsos respirométricos de acetato e de propionato no licor misto dos sistemas RBS1, RBS2 e RBS3. As barras de erro representam os desvios padrão dos respectivos conjuntos de dados.

Nota-se facilmente que as taxas específicas de consumo de oxigênio dos lodos dos reatores são mais elevadas com acetato do que com propionato como substrato, corroborando a existência de organismos acumuladores de polifosfato e a dificuldade de outros grupos de organismos competidores (e.g, GAO) em metabolizar propionato. Ademais, os valores médios de TCOe com os licores dos reatores avaliados, nos pulsos com os substratos testados, indicaram seguir uma distribuição normal ( $p$-valor > 0,05), de acordo com os testes de Shapiro-Wilk realizados.

Então, testes de ANOVA evidenciaram diferenças significativas ( $p$-valor $<0,05$ ) somente entre os valores TCOe dos lodos após pulso com o 
propionato. Por esse motivo, testes de Bonferroni foram, adicionalmente, realizados e revelaram haver uma diferença significativa ( $p$-valor $<0,05$ ) da TCOe do licor do RBS2 em relação aos valores dos reatores RBS1 e RBS3, após pulso respirométrico com propionato, sugerindo uma atividade metabólica mais intensa das DPAO II na presença deste substrato, sugerindo um provável predomínio desses organismos entre os acumuladores de polifosfato presentes nos lodos de sistemas de remoção avançada de fósforo, como observado por Bassin et al. (2012), quando o ciclo de operação apresenta etapas com condições anóxicas.

\section{CONCLUSÕES}

As biomassas utilizadas nos reatores de bateladas sequenciais mantidos em condições experimentais controladas, particularmente a idade do lodo, a temperatura abaixo de $20^{\circ} \mathrm{C}$ e o $\mathrm{pH}$ na faixa de 7,0 a 8,0, e seguindo as estratégias propostas neste estudo, produziram licores mistos constituídos por quantidades relevantes de organismos acumuladores de polifosfato (PAO e DPAO), os quais foram muito satisfatórios na remoção de matéria orgânica (acetato), desfosfatação e, nos sistemas com fases anóxicas (após fase anaeróbia), na desnitrificação do efluente sintético periodicamente alimentado nos reatores.

Os três RBS propostos e estudados, em escala de bancada, foram capazes de remover quase $90 \%$ de matéria orgânica (em termos de DQO) e mais de $70 \%$ do ortofosfato solúvel presente no sistema (em termos de fósforo). Ademais, os dois reatores com etapas reacionais anóxicas removeram mais de $80 \%$ do nitrogênio em excesso (como nitrito ou nitrato) adicionado ao meio.

Os resultados também indicaram que o reator menos eficiente entre os estudados para remoção de fósforo foi o conduzido com fase anóxica e nitrato como aceptor de elétrons (RBS3). Esse reator também foi o que apresentou o maior quantitativo de lodo produzido, o que pode representar custos adicionais no descarte desse material. Assim, o uso do nitrito como aceptor de elétrons pode ser mais efetivo em sistemas biológicos avançados de remoção de fósforo com etapas anóxicas e em condições similares às adotadas neste trabalho.

Os reatores com fase aeróbia e fase anóxica, respectivamente oxigênio e nitrito como aceptores de elétrons, foram estatisticamente equivalentes em desempenho (remoção de matéria orgânica e de fosforo) e da produção de biomassa. Contudo, considerando os custos adicionais inerentes à aeração do reator, particularmente quando o efluente a tratar já apresentar níveis razoáveis de nitrito, pode-se afirmar que o RBS com fase anóxica (com nitrito como aceptor de elétrons), operado nas condições aqui testadas, é o sistema mais viável para uma remoção satisfatória de fósforo e matéria orgânica de efluentes.

Finalmente, as duas ferramentas de avaliação e o parâmetro cinético, baseados em ensaios respirométricos e físico-químicos e utilizados neste estudo, foram satisfatórios na avaliação da ocorrência e predominância de organismos acumuladores de polifosfato no lodo dos RBS testados, corroborando também os resultados de desempenho observados e as influências dos aceptores de elétrons utilizados no processo.

\section{AGRADECIMENTOS}

Os autores agradecem ao suporte financeiro e de infraestrutura do Laboratório de Controle Ambiental do campus Limoeiro do Norte do Instituto Federal de Educação, Ciência e Tecnologia do Ceará e à Fundação Cearense de Apoio ao Desenvolvimento Científico e Tecnológico (FUNCAP) pela bolsa de mestrado concedida. 


\section{CONTRIBUIÇÃO DOS AUTORES}

Todos os autores contribuíram de forma igualitária.

\section{REFERÊNCIAS}

APHA. Standard Methods for the Examination of Water and Wastewater. $23^{\text {rd }}$ edition. Washington, DC: APHA; AWWA; WEF, 2017.

BASSIN, J. P.; KLEEREBEZEM, R.; DEZOTTI, M. W. C.; VAN LOOSDRE$\mathrm{CHT}$, M. C. M. Simultaneous nitrogen and phosphate removal in aerobic granular sludge reactors operated at different temperatures. Water Resources, v. 46, p. 3805-3816, 2012. https://doi. org/10.1016/j.watres.2012.04.015

BOWES, M. J.; JARVIE, H. P.; HALLIDAY, S. J.; SKEFFINGOTON, R. A.; WADE, A. J.; LOEWENTHAL, M.; GOZZAD, E.; NEWMAN, J. R.; PALMER-FELGATE, E. J. Characterizing phosphorus and nitrate inputs to a rural river using high-frequency concentration-flow relationships. Science of The Total Environment, v. 511, p. 608-620, 2015. https://doi.org/10.1016/j.scitotenv.2014.12.086

BUNCE, J, T., NDAM, E., OFITERO, I, D., MOORE, A., GRAHAM, D, D. A Review of Phosphorus removal technologies and their applicability to small-scale domestic wastewater treatment systems. Frontiers in Environmental Science, v. 6, p. 8, 2018. https://doi. org/10.3389/fenvs.2018.00008

CATUNDA, S. Y. C.; DEEP, G. S.; VAN HAANDEL, A. C.; FREIRE, R. C. $S$. Fast on-line measurement of the respiration rate in activated sludge systems. IEEE Instrumentation and measurement technology conference Bruxelas, Bélgica, p. 4-6, 1996. https://doi. org/10.1109 / IMTC.1996.507585

COAT, E. R., DOBROTH, T. Z., BRINKMAN, K. C. EBPR using crude glycerol: assessing process resiliency and exploring metabolic anomalies. Water Environment Research, v. 87, p. 68-79, 2015. https://doi.org/10.2175/106143014X14062131179113

COSTA, R. H. R.; VILLAFRANCA, B. M.; VOLTOLINI, C. A.; GUIMARÃES, L. B.; HOFFMANN, H.; VELHO, V. F.; MOHEDANO, R. A. Effectiveness of phosphorus removal in an SBR using co-precipitation with ferric chloride, and its effects on microbial activity. Brazilian Journal of Chemical Engineering, v. 36 (2), p. 785-795, 2019. https://doi.org/10.1590/0104-6632.20190362s20180378

CYDZIK-KWIATKOWSKA, A.; ZIELINSKA, M. Bacterial communities in full-scale wastewater treatment systems. World Journal of Microbiology and Biotechnology, v. 32, p. 66-74, 2016. https://doi. org/10.1007/s11274-016-2012-9

DERKS, Y. M. Uso da respirometria para avaliar a influência de fatores operacionais e ambientais sobre a cinética de nitrificação. 2007. 103 f. Dissertação (Mestrado). Campina Grande: Universidade Federal de Campina Grande, 2007. http://dspace. sti.ufcg.edu.br:8080/jspui/handle/riufcg/8890
FERREIRA, A. L. T. S. Remoção biológica simultânea de fósforo e nitrogênio de esgoto sanitário em reatores sequenciais em batelada. 2014. 164p. Tese (Doutorado em Engenharia Civil). Recife: Universidade Federal de Pernambuco, 2014. https://repositorio. ufpe.br/handle/123456789/17544

FIELD, A. Descobrindo a estatística usando o SPSS. $5^{\mathrm{a}}$ ed. Porto Alegre: Penso, 2020.

GE, J., MENG, X., SONG, Y., TERRACCIANO, A. Effect of phosphate releasing in activated sludge on phosphorus removal from municipal wastewater. Journal of Environmental Sciences, v. 67, p. 216-223, 2018. https://doi.org/10.1016/j.jes.2017.09.004

GEYER, C. J. Stat $\mathbf{5 1 0 2}$ Notes: Nonparametric tests and confidence intervals. In: Statistics 5102 (Geyer) Spring 2003, University of Minnesota, Twin Cities, 2003. Disponível em: <http://www. stat.umn.edu/geyer/old03/5102/notes/rank.pdf>. Acessado em: 31 mar. 2020.

LEE, S. H.; JUNG, S. G.; PARK, S. M.; LEE, B. D. Evaluation of the tributaries by influence index on the mid-lower portion of the Nakdong River basin. Environmental Engineering Research, v. 23, p. 150158, 2018. https://doi.org/10.4491/eer.2017.067

LI, C.; LIU, S.; MA, T.; ZHENG, M.; NI, J. Simultaneous nitrification, denitrification and phosphorus removal in a sequencing batch reactor (SBR) under low temperature. Chemosphere, v. 229, p. 132141, 2019. https://doi.org/10.1016/j.chemosphere.2019.04.185

LIU, Y.; HU, T.; SONG, Y.; CHEN, H.; LV, Y. Heterotrophic nitrogen removal by Acinetobacter sp. $Y 1$ isolated from coke plant wastewater. Journal of Bioscience and Bioengineering, v. 120, p. 549554, 2015. https://doi.org/10.1016/j.jbiosc.2015.03.015

MAHARDIKA, D.; PARK, H. S.; COO, K. H. Ferrihydrite-impregnated granular activated carbon (FHaGAC) for efficient phosphorus removal from wastewater secondary effluent. Chemosphere, v. 207, p. 527-533, 2018. https://doi.org/10.1016/j.chemosphere.2018.05.124

MELQUIADES, R. M. L.; SANTOS, E. V.; HERALDO FILHO, A. S.; VAN HAANDEL, A. C.; SOUSA, J. T. Ferramentas para identificação da predominância de organismos acumuladores de fósforo (PAO) em sistemas de lodo ativado. In: Congresso Brasileiro de Engenharia Sanitária, 29, 2017, São Paulo. Rio de Janeiro: ABES, 2017. Anais...

METCALF, L. \& EDDY, H. P. Tratamento de Efluentes e Recuperação de Recursos. $5^{\mathrm{a}}$ ed. Porto Alegre: AMGH, 2016. ISBN: 0073401188.

MIRANDA, A. C. Influência da concentração de lodo sobre a capacidade de oxigenação do aerador e a influência da concentração do sal sobre a capacidade metabólica do lodo. 2012. 90 f. Dissertação (Mestrado em Engenharia Civil e Ambiental). Campina Grande: Universidade Federal de Campina Grande, 2009. http://dspace.sti.ufcg.edu.br:8080/jspui/handle/riufcg/10298

OEHMEN, A.; YUAN, Z.; BLACKALL, L. L.; KELLER, J. Comparison of acetate and propionate uptake by polyphosphate accumulating 
organisms and glycogen accumulating organisms. Bioresource Technology, v. 91, p. 162-168, 2005. https://doi.org/10.1002/ bit. 20500 .

OEHMEN, A.; SAUNDERS, A. M.; VIVES, M. T.; YUAN, Z.; KELLER, J. Competition between polyphosphate and glycogen accumulating organisms in enhanced biological phosphorus removal systems with acetate and propionate as carbon sources. Journal of Biotechnology, v. 123 (1), p. 22-32, 2006a. https://doi.org/10.1016/j. jbiotec.2005.10.009

OEHMEN, A.; ZENG, R. J.; SAUNDERS, M. A.; BLACKALL, L. L.; KELLER, J.; YUAN, Z. Anaerobic and aerobic metabolism of glycogen-accumulating organisms selected with propionate as the sole carbon source. Microbiology, v. 152, p. 2767-2778, 2006b. https://doi.org/10.1099/mic.0.28065-0

OEHMEN, A.; LEMOS, P. C.; CARVALHO, G.; YUAN, Z.; KELLER, J.; BLACKALL, L. L.; REIS, M. A. M. Advances in enhanced biological phosphorus removal:From micro to macro scale. Water Resources, v. 41, p. $2271-2300,2007$. https://doi.org/10.1016/j.watres.2007.02.030

PANSWAD, T.; DOUNGCHAI, A.; ANOTAI, J. Temperature effect on microbial community of enhanced biological phosphorus removal system. Water Research, v. 37 (2), p. 409-415, 2003. https://doi. org/10.1016/S0043-1354(02)00286-5

RANJAN, P.; BHUNIA, R. P.; DASH, R. R. Simultaneous removal of nitrogen and phosphorous from domestic wastewater using Bacillus cereus GS-5 strain exhibiting heterotrophic nitrification, aerobic denitrification and denitrifying phosphorous removal. Bioresource Technology, v. 244, p. 484-495, 2017. https://doi. org/10.1016/j.biortech.2017.07.186

SANTOS, E. V. M. Estratégias para predominância de organismos acumuladores de fósforo em sistemas de lodo ativado e respirometria aplicada à biodesfosfatação. 2014. 267 f. Tese (Doutorado). Campina Grande: Universidade Federal de Campina Grande, 2014. http://tede.bc.uepb.edu.br/jspui/handle/tede/2247.

SILVA FILHO, H. A. Nitrificação em Sistemas de Lodo Ativado. 2009. 134 f. Dissertação (Mestrado em Engenharia Civil e Ambiental). Campina Grande: Universidade Federal de Campina Grande, 2009. http://dspace.sti.ufcg.edu.br:8080/jspui/handle/ riufcg/10576

SMOLDERS, G. J. F., VAN LOOSDRECHT, M. C. M., HEINEN, J. J. Model of the anaerobic metabolism of the biological phosphorus removal process: stoichiometry and $\mathrm{pH}$ influence. Biotechnology and Bioengineering, v. 43, p. 461-470, 1994. https://doi. org/10.1002/bit.260430605

STENSEL, H. D. Principles of biological phosphorus removal. In: Phosphorus and nitrogen removal from municipal wastewater: Principles and practice. SEDLAK, R. I. $2^{\mathrm{a}}$ ed. New York: Lewis publishers. p. 141-163, 1991. . https://doi. org/10.1201/9780203743546
TAYÀ, C.; GARLAPATI, V. K.; GUISASOLA, A.; BAEZA; J. A. The selective role of nitrite in the PAO/GAO competition. Chemosphere, v. 93, p. 612-618, 2013. https://doi.org/10.1016/j.chemosphere.2013.06.006.

TRIOLA, M. F. Introdução à Estatística. $12^{\mathrm{a}}$ ed. Rio de Janeiro: LTC, 2017.

VAN HAANDEL, A. C.; CATUNDA, P. F. C. Determinação da taxa de consumo de oxigênio em sistemas de tratamento de esgotos. Revista de Engenharia Sanitária, v. 21 (4), p. 481-488, 1982.

VAN HAANDEL, A. C.; MARAIS, G. 0 comportamento do sistema de lodo ativado: teoria e aplicações para projetos e operações. Campina Grande: EPGRAF, 1999. BR67.1; 628.3,72.47608/2006.

VON SPERLING, M. Activated sludge and aerobic biofilm reactors. [Volume 5]. London: IWA publishing, 2007. https://doi. org/10.2166/9781780402123

WANG, D. B.; LI, X. M.; YANG, Q.; ZENG, G. M.; LIAO, D. X.; ZHANG, J. Biological phosphorus removal in sequencing batch reactor with single-stage oxic process. Bioresource Technology, v. 99 (13), p. 5466-5473, 2008. https://doi.org/10.1016/j.biortech.2007.11.007.

WANG, L; LIU, L; ZHENG, B. Eutrophication development and its key regulating factors in a water-supply reservoir in North China. Journal of Environmental Sciences, v. 25, p. 962-970, 2013. https://doi.org/10.1016/S1001-0742(12)60120-X

WANG, X.; WANG, S.; XUE, T.; LI, B.; DAI, X.; PENG, Y. Treating low carbon/nitrogen $(\mathrm{C} / \mathrm{N})$ wastewater in simultaneous nitrification-endogenous denitrification and phosphorous removal (SNDPR) systems by strengthening anaerobic intracellular carbon storage. Water Resources, v. 77, p. 191-200, 2015. https://doi.org/10.1016/j.watres.2015.03.019

YANG, Y., ZHANG, L., SHAO, H., ZHANG, S., GU, P., PENG, Y. Enhanced nutrients removal from municipal wastewater through biological phosphorus removal followed by partial nitritation/anammox. Frontiers of Environmental Science \& Engineering, v. 11 (2), p. 8-13, 2017. https://doi.org/10.1007/s11783-017-0911-0

ZAMAN, M.; KIM, M.; NAKHLA, G.; SINGH, A.; YANG, F. Enhanced biological phosphorus removal using thermal alkaline hydrolyzed municipal wastewater biosolids. Journal of Environmental Sciences, v. 86, p. 164-174, 2019. https://doi.org/10.1016/j. jes.2019.05.025

ZHAO, J.; WANG, X.; LI, X.; JIA, S.; WING, Q.; PENG, Y. Improvement of partial nitrification endogenous denitrification and phosphorus removal system: Balancing competition between phosphorus and glycogen accumulating organisms to enhance nitrogen removal without initiating phosphorus removal deterioration. Bioresource Technology, v. 281, p. 382-391, 2019. https://doi. org/10.1016/j.biortech.2019.02.109 